\title{
Modified balloon-stent kissing technique avoid side-branch compromise for simple true bifurcation lesions
}

Wen-Bo Qu, Wei Zhang, Jun-Yan Liu, Fan Zhang, Shuai-Nan Mu, Sheng-Ming Zhang, Hao Tang, Xi-Qian Liu, Xue-Qi Li and Bing-Chen Liu* (D)

\begin{abstract}
Background: Coronary bifurcation remains one of the most challenging lesion subsets in interventinal cardiology. Provisional stenting (PS) is the dominate technique for bifurcation lesions, but the key problem is the deterioration of side branch. Balloon-stent kissing technique (BSKT) as a new systematic approach which is based on modified jailed balloon technique is applied to improve the procedure success. In our center, we proposed a modified balloon-stent kissing technique(M-BSKT), which routine usage of proximal optimizing technique (POT) after rewiring was added as an optimization step to BSKT. Thus, whether M-BSKT for addressing simple true coronary bifurcation lesions can provide more benefits in intra-operation effect and long term outcomes is still unknown.
\end{abstract}

Methods: A cohort of 120 consecutive patients underwent Percutaneous Coronary Intervention (PCI) with simple true coronary bifurcation lesions satisfied the criteria were included in this retrospective, single-center registry. To assemble a cohort with similar baseline characteristics, a 1:1 propensity-matched score was used. The primary outcomes were the rate of device and procedural success, the situation of side branch (SB) after main vessel (MV) inflation and the complications during intra-operative. The secondary outcomes were the clinical prognosis at 12 months such as rehospitalization for unstable angina and MACEs.

Results: Before propensity matching, there were no significant differences in primary and secondary outcomes between two groups. After propensity-matched was used, 68 patients with similar propensity scores were included. At immediate procedural, M-BSKT was associated with a lower risk of SB deterioration and the application of final kissing balloon inflation $(F K B I)[P=0.036]$. For ACS patients, besides the significant differences of immediate SB deterioration $[P=0.014]$ and FKBI application $[P=0.033]$, the incidence of TIMl flow $<3$ in the PS was statistically significant higher than M-BSKT $[P=0.042]$. The prognosis at 12 months such as rehospitalization for unstable angina and MACEs were similar for two groups $[P=0.613]$.

Conclusion: These observations prove that the M-BSKT enables side branch to be better protected in simple true bifurcation lesions, by a narrow margin. It may improve the angiographic outcomes about side branch deterioration and final kissing balloon performing compared with PS, especially in ACS patients. However, long-term clinical outcomes did not differ between patients treated for M-BSKT and PS at 12 months.

Keywords: Modified balloon-stent kissing technique, Provisional stenting, Bifurcation lesions, Percutaneous coronary intervention, Side branch deterioration, Acute coronary syndrame

\footnotetext{
* Correspondence: lixuegi@medmail.com.cn; liubingchen111@126.com

Department of Cardiology, The Fourth Affiliated Hospital of Harbin Medical University, Harbin, Heilongjiang 150086, People's Republic of China
}

(c) The Author(s). 2019 Open Access This article is distributed under the terms of the Creative Commons Attribution 4.0 International License (http://creativecommons.org/licenses/by/4.0/), which permits unrestricted use, distribution, and reproduction in any medium, provided you give appropriate credit to the original author(s) and the source, provide a link to the Creative Commons license, and indicate if changes were made. The Creative Commons Public Domain Dedication waiver (http://creativecommons.org/publicdomain/zero/1.0/) applies to the data made available in this article, unless otherwise stated. 


\section{Background}

Coronary bifurcations are frequent and account for approximately $20 \%$ of all percutaneous coronary interventions [1]. Nonetheless, they represent one of the remaining challenges in interventional cardiology and the uniform strategy is still the subject of substantial debate in terms of a lower procedural success rate,a higher risk of procedural complications, and increasing rates of long-term adverse cardiac events [2].

During stent implantation, provisional stenting (PS) was often performed, where a conventional guide wire was inserted to the side branch (SB) before implanting stent to the main vessel (MV), if it wasn't effective, then the SB could be treated. It has been widely accepted as the gold standard for its lower risks of major adverse cardiac events (MADEs), death, myocardial infarction (MI), and target vessel revascularization (TVR) in the majority of bifurcation lesions [3-11].

Whereas, simple strategy may shift the carina to the SB and induce a stenosis after stenting of MV. Major SB occlusion after MV stenting is one of the most serious complications closely associated with cardiac death and MI, and it may be the major reason why operators prefer more aggressive strategy in the bifurcation lesions [12-15]. Therefore, to prevent from carina displacement-the basic mechanism of side branch compromise during bifurcation percutaneous coronary intervention, several novel stenting systems for bifurcation lesions have been developed [16, 17].

The main purpose of this study was to perform a novel SB protection technique called Modified balloon-stent kissing technique (M-BSKT), which was based on balloon-stent kissing technique that was first proposed by Jin Z [18]. Routine usage of proximal optimizing technique (POT) after rewiring could make the malapposition in the stented MV segment completely corrected while maintaining perfect arterial circularity and achieving effective modification of physiological anatomy [19]. With this improvement, we modified balloon-stent kissing technique and discussed the advantage compared to other $\mathrm{SB}$ protection techniques such as PS.

\section{Methods}

\section{Study population}

From January 2015 to July 2017, a cohort of 120 consecutive patients who defined as true bifurcation lesions undergoing PCI were collected at The Fourth Affiliated Hospital of Harbin Medical University, Harbin, China.

All the patients enrolled in our study population were patients with simple true bifurcation lesions (excluding the complex bifurcation lesions [20]). The study was approved by the institutional review board, and informed consents for participation in the trial were obtained from all patients.

Data collection and monitoring were performed by an independent third-party contract research organization. The executive committee met regularly in person to monitor all aspects of the conduct of the trial.

\section{Inclusion criteria}

(1) Patients were eligible for the studies if they were 18 to75 years old with true coronary bifurcation lesions undergoing PCI

(2) The true bifurcation lesion consisted at least one major SB, bifurcation classifications were made according to Medina classification [21]. Medina 1,1,1 1,0,1 and 0,1,1 coronary bifurcation lesions with an SB diameter $\geq 2.0 \mathrm{~mm}$ based on visual estimation were included in the training and study groups

\section{Exclusion criteria}

(1) The bifurcation lesion was categorized as complex bifurcation lesions according to the DEFINITION [20], defined as Medina 1,1,1 and 0,1,1 coronary bifurcation lesions with each major criterion (left main vessel with ostial SB lesion length $\geq 10 \mathrm{~mm}$ and diameter stenosis (DS) $\geq 70 \%$; non-left main vessel with ostial $\mathrm{SB}$ lesion length $\geq 10 \mathrm{~mm}$ and DS $\geq 90 \%$ ) plus any 2 minor criteria (moderate to severe calcification; multiple lesions; bifurcation angle $>45$; main vessel $R V D<2.5 \mathrm{~mm}$; thrombuscontaining lesions; MV lesion length $\geq 25 \mathrm{~mm}$ )

(2) Subject with renal failure (serum creatinine $>2.0$ $\mathrm{mg} / \mathrm{dl}$ )

(3) Subject exhibited severe left ventricular dysfunction (left ventricular ejection fraction $<35 \%$ )

(4) Subject with a serious comorbidity or with life expectancy $<1$ year

(5) Subject exhibited contraindications to aspirin or clopidogrel

\section{Procedure and outcomes}

According to the ratio of $1: 2$, we randomly gathered patients with simple true bifurcation lesions to M-BSKT $(n=40)$ and PS $(n=80)$. The study patients in both groups received the same routine preparation with antiplatelet and antithrombotic medications based on the current guidelines. Coronary angioplasty was performed in conventional manner, and the interventional strategy and instrumentation used were at the discretion of the interventional cardiologists. Decisions on treatment strategy for bifurcation lesions were attempted according to the randomization process by individual operators. 
Provisional stenting (jailed wire) technique The recommended strategy is to wire in both MV and SB, while the lesion preparation is made at the operator's discretion. The MV is stented with jailed wire protecting in SB. Proximal Optimization Technique (POT) is mandated to achieve good apposition of the proximal MV stent after the SB is rewired with or without rescue final kissing (Indications are shown below). The wire in SB will not be removed until the POT is completed.

Modified balloon-stent kissing technique The initial technique has been detailed in previous study [18]. The M-BSKT used at our institution is detailed in Fig. 1 and shown angiographically in Fig. 2: To be brief, vessel wiring and lesion preparation are the same as the jailed wire technique. A balloon that is appropriately sized to approximate or smaller than the reference vessel diameter of SB (generally $1.5-2.5 \mathrm{~mm}$ ) is advanced into the SB. A stent is then advanced into correct position over the target lesion in the MV. Adequate length of balloon is advanced into SB to project the ostium. Then the jailed SB balloon is first inflated to low pressure (6-8 atm), meanwhile, the stent in MV is deployed to nominal pressure $(8-10 \mathrm{~atm})$ (Fig. $2 \mathrm{~b}-\mathrm{c})$. The SB balloon is removed and the wire remains in SB. The stent balloon is then fully expanded to moderate or high pressure as clinically indicated for the optimization of the stent full expansion at the distal MV, and to correct any stent deformation as a result of the SB balloon inflation (Fig. 2d). If the $\mathrm{SB}$ is not compromised, the $\mathrm{SB}$ will be rewired followed by POT (Fig. 2e) that achieve effective modification of physiological anatomy. Final result was good in showing case (Fig. 2f).

The SB is not further treated both in PS and M-BSKT groups unless there is threatened SB closure, severe ostial pinching of SB (>90\%), TIMI flow grade decrease in $\mathrm{SB}$, or $\mathrm{SB}$ dissection greater than type A. If one of these criteria exists, the SB will be rewired and a final kissing balloon inflation (FKBI) will be undertaken with anatomically appropriate sizing for each vessel. A bailout stent in side branch is implanted only in case of dissection or significant flow impairment after FKBI. Finally, the re-POT strategy is mandated to achieve optimization apposition of the

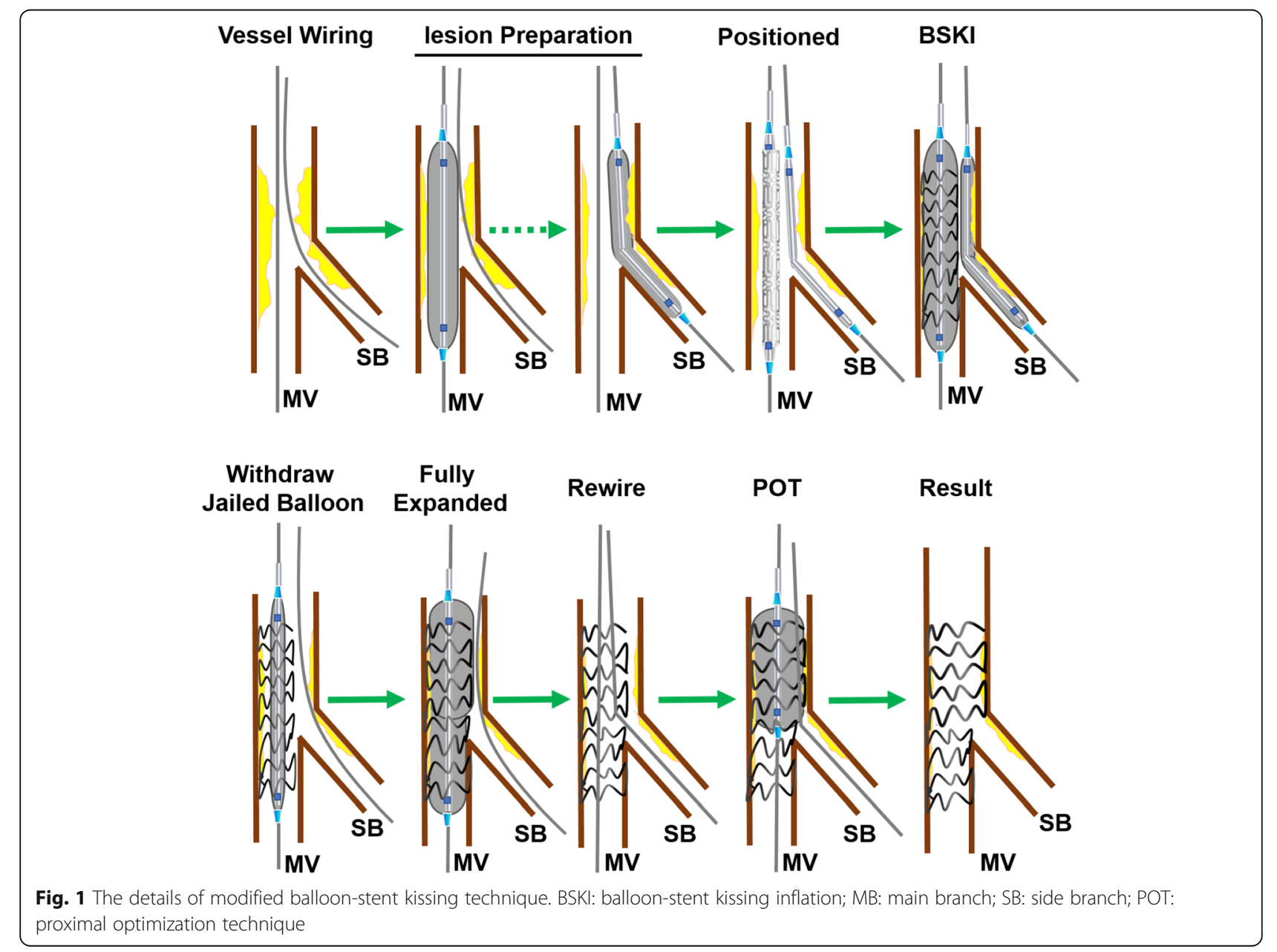



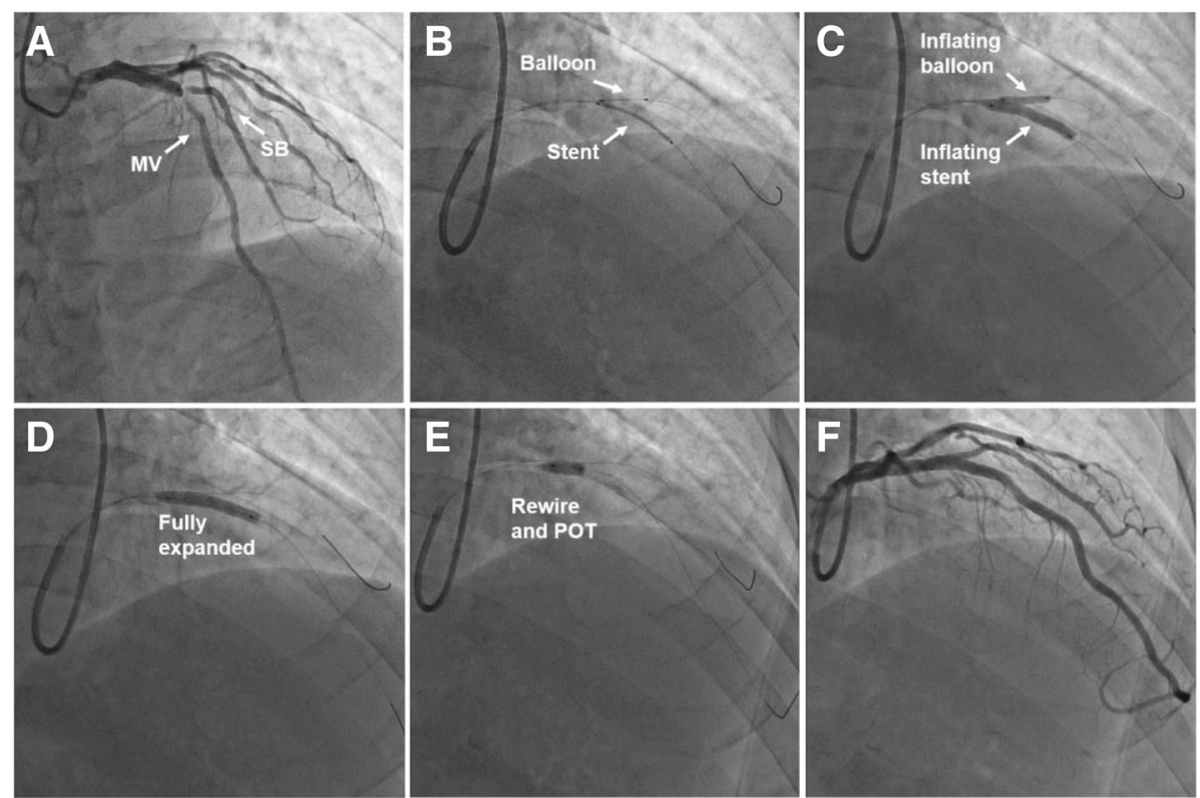

Fig. 2 Coronary angiography illustrating the modified balloon-stent kissing technique. a: The angiogram demonstrates severe stenosis at simple true bifurcation segment (Medina 1,1,1). b: A balloon that is sized to the reference vessel diameter of SB $(2.5 \mathrm{~mm})$ is advanced into the SB. A stent $(3.5 \mathrm{~mm})$ is then advanced into correct position over the target lesion in the MV. c: The jailed SB balloon is inflated to 6 atm, meanwhile, the stent in MV is deployed to $8 \mathrm{~atm}$. $\mathbf{d}$ : The stent balloon is fully expanded to $12 \mathrm{~atm}$. e: The SB is rewired followed by POT (4.0 mm non-compliant

balloon). $\mathbf{f}$ : No residual stenosis both in MV and SB in final angiogram

proximal MV stent. In addition, it needs to be emphasized that the MV stent should be sized according to the distal main vessel diameter to prevent irreversible carina shift, and the non-compliant balloon should be chosen for POT and sized according to the Murray's law.

At postprocedure, all patients received aspirin (100 $\mathrm{mg} / \mathrm{d})$ indefinitely, and clopidogrel $(75 \mathrm{mg} / \mathrm{d})$ would be recommended for at least 12 months. The administration of glycoprotein IIb/IIIa inhibitors was performed at the discretion of the operator.

\section{Follow-up}

Clinical follow-up was performed by physicians via medical chart review or telephone interview at 12 months. Adverse events were monitored throughout the entire study period. Angiographic re-evaluation was planned after 1 year. The information of 120 patients was eventually collected.

\section{Primary and secondary endpoint(s)}

The primary endpoints of the study were the rates of device and procedural success (lesion success without the occurrence of in-hospital major adverse cardiac events (MACEs) such as death, myocardial infarction (MI), emergent coronary artery bypass graft (CABG), clinically driven target lesion revascularization (TLR)), the total numbers of FKBI used (because of the SB ostial pinching $\geq 90 \%$ and the TIMI flow $<3$ after the MB stenting), the incidence of SB occlusion(after MV stent inflation) and the probability of balloons and wires damaged or even broken during their removal.

Secondary endpoints were a composite of the rehospitalization rate due to angina pectoris and MACEs at 12 months (defined as cardiac death, MI, and any TLR by PCI or CABG).

\section{Statistical analysis}

Descriptive statistics were presented as percentages for categorical variables. Baseline characteristics were compared using a Chi-square or Fisher exact test for categorical variables and a Mann-Whitney $U$ test for continuous variables because of they not compared to normal distribution. For non-normally distributed variables, median and IQR were used. In addition to conventional multivariate analysis, we constructed a propensity score within $\mathrm{a} \pm 0.2$ caliper range for adjustment and matching using $\mathrm{R}$ package matching. Matched categorical and continuous variables were presented same as before. A $P$ value of $<0.05$ was considered to represent a statistically significant difference. All the statistical models were performed using R Programming Language.

\section{Results}

\section{Baseline characteristics}

Between January 2015 and July 2017, a total of 120patients with true coronary bifurcation lesions satisfied all 
inclusion/exclusion criteria undergoing PCI were enrolled and ramdomly assigned to $\operatorname{M-BSKT}(n=40)$ and $\operatorname{PS}(n=80)$. All patients were followed up for 12 months. There were no significant differences between the two groups for patient's baseline clinical characteristics (Table 1) and lesion characteristics (Table 2),respectively.

\section{Procedural outcomes}

The immediate procedural and clinical outcomes are displayed in Table 3. The operation successful rate was $100 \%$ in each group. The study showed no statistically significant clinical impact of SB deterioration [M-BSKT $n=7(17.5 \%) ;$ PS $n=28(35.0 \%) ; P=0.076]$ in 2 groups (Fig. 3a). The rates of SB TIMI flow $<3$ were $7.5 \%$ and $15 \%[P=0.38]$, severe $\mathrm{SB}$ ostial pinching $(\geq 90 \%)$ were $10 \%$ and $17.5 \%[P=0.412]$, in the M-BSKT versus the PS groups, respectively. Besides, 2 patients' SB occluded immediately in PS, and the other patients whose SB damaged underwent FKBI [M-BSKT $n=7(17.5 \%)$; PS $n=26(32.5 \%)$; $P=0.129$ ] (Fig. 3b). SB flow was restored spontaneously in 2 groups [M-BSKT $\mathrm{n}=7(17.5 \%) ;$ PS $n=23(28.8 \%) ; P=$ 0.263]. The use of bailout SB stenting was $2.5 \%$ of the PS group $[\mathrm{n}=2 ; P=0.552]$. Compared with M-BSKT, 5 patients'SB in PS were inevitable occluded despite all SB had jailed wire, even though 3 of them underwent FKBI [M-BSKT $n=0$; PS $n=5(6.3 \%)$ ); $P=0.168$ ]. All the wires and balloons were not entrapped during the operations.

\section{Clinical outcomes at 12 months}

Long-term clinical outcomes did not differ between patients treated with M-BSKT and PS at 12 months $[P=$ $0.558]$. The cumulative rehospitalization for unstable angina was similar between M-BSKT [3(7.5\%)] and PS $[\mathrm{n}=4(5.0 \%)]$. The cumulative 1-year MACEs was only occurred in PS group [n=3(3.8\%)] (Table 3 and Fig. 4).

\section{Propensity score matching}

We conducted propensity score matching by using $\mathrm{R}$ package matching and matched patients within $\mathrm{a} \pm 0.2$ caliper range. The following 15 preoperative variables were considered in the stepwise logistic regression multivariable analysis: male, age, hypertension, diabetes mellitus, hyperlipidemia, smoke, prior CABG, prior PCI, prior $\mathrm{MI}$, chronic kidney disease, diagnosis at $\mathrm{PCI}$, Troponin I (TNI), Creatine Kinase-MB (CK-MB), the location of bifurcation lesions and Medina classification.

A matched pair of 34 M-BSKT and 34 PS patients was identified. Patient characteristics were well matched

Table 1 Baseline Characteristics

\begin{tabular}{|c|c|c|c|c|c|c|}
\hline & \multicolumn{3}{|c|}{ pre propensity-matched analysis } & \multicolumn{3}{|c|}{ post propensity-matched analysis } \\
\hline & M-BSKT & PS & $P$ Value & \multirow{2}{*}{$\begin{array}{l}\text { M-BSKT } \\
n=34\end{array}$} & \multirow{2}{*}{$\begin{array}{l}P S \\
n=34\end{array}$} & \multirow[t]{2}{*}{$P$ Value } \\
\hline & $n=40$ & $\mathrm{n}=80$ & & & & \\
\hline Male & $30(75 \%)$ & $62(77.5 \%)$ & 0.939 & $25(73.5 \%)$ & $26(76.5 \%)$ & 1 \\
\hline Hypertension & $28(70 \%)$ & $42(52.5 \%)$ & 0.102 & $22(64.7 \%)$ & $21(61.8 \%)$ & 1 \\
\hline Diabetes Mellitus & $14(35 \%)$ & $19(23.8 \%)$ & 0.202 & $10(29.4 \%)$ & $10(29.4 \%)$ & 1 \\
\hline Hyperlipidemia & $14(35 \%)$ & $25(31.3 \%)$ & 0.836 & $13(38.2 \%)$ & $10(29.4 \%)$ & 0.608 \\
\hline Smoker & $15(37.5 \%)$ & $39(48.8 \%)$ & 0.331 & $14(41.2 \%)$ & $20(58.8 \%)$ & 0.225 \\
\hline prior $C A B G$ & $1(2.5 \%)$ & 0 & 0.333 & 0 & 0 & NS \\
\hline prior $\mathrm{PCl}$ & $6(15 \%)$ & $14(17.5 \%)$ & 0.931 & $5(14.7 \%)$ & $3(8.8 \%)$ & 0.709 \\
\hline prior Ml & $4(10 \%)$ & $9(22.5 \%)$ & 1 & $2(5.9 \%)$ & $1(2.9 \%)$ & 1 \\
\hline Chronic kidney disease & $1(2.5 \%)$ & $2(2.5 \%)$ & 1 & $1(2.9 \%)$ & 0 & 1 \\
\hline \multicolumn{7}{|l|}{ Diagnosis at $\mathrm{PCl}$} \\
\hline STEMI & $3(7.5 \%)$ & $11(13.8 \%)$ & 0.464 & $2(5.9 \%)$ & $4(11.7 \%)$ & 0.577 \\
\hline NSTEMI & $9(22.5 \%)$ & $22(27.5 \%)$ & & $6(17.6 \%)$ & $8(23.5 \%)$ & \\
\hline Angina & $28(70 \%)$ & 47 (58.8\%) & & $26(76.5 \%)$ & 22 (64.7\%) & \\
\hline Age & 62 & 59.5 & 0.255 & 62 & 59 & 0.368 \\
\hline$I Q R$ & 12 & 13 & & 13.5 & 10.5 & \\
\hline $\mathrm{TNI}$ & 0.01 & 0.01 & 0.487 & 0.01 & 0.01 & 0.533 \\
\hline $\mathrm{IQR}$ & 0.068 & 0.187 & & 0.021 & 0.21 & \\
\hline CK-MB & 14 & 15 & 0.397 & 14 & 15 & 0.1 \\
\hline $\mathrm{IQR}$ & 4.25 & 6.25 & & 4 & 5.75 & \\
\hline
\end{tabular}

Data are presented as median (interquartile range) for continuous variables and $\mathrm{n}$ (\%) for categorical variables. Chi-square or Fisher exact test for categorical variables and a Mann-Whitney $\mathrm{U}$ test for continuous variables. CABG, coronary artery bypass graft; $\mathrm{PCl}$, percutaneous coronary intervention; $\mathrm{MI}$, myocardial infarction; STEMI, ST-segment elevation myocardial infarction; NSTEMI, non-ST-segment elevation myocardial infarction; TNI, Troponin I; CK-MB, Creatine Kinase-MB 
Table 2 Lesion characteristics

\begin{tabular}{|c|c|c|c|c|c|c|c|}
\hline & & \multicolumn{3}{|c|}{ pre propensity-matched analysis } & \multicolumn{3}{|c|}{ post propensity-matched analysis } \\
\hline & & M-BSKT & PS & $P$ Value & \multirow{2}{*}{$\begin{array}{l}\text { M-BSKT } \\
n=34\end{array}$} & PS & \multirow[t]{2}{*}{$P$ Value } \\
\hline & & $\mathrm{n}=40$ & $\mathrm{n}=80$ & & & $n=34$ & \\
\hline \multirow[t]{5}{*}{ Location } & LAD-D1 & $27(67.5 \%)$ & $56(70.0 \%)$ & 0.481 & $26(76.5 \%)$ & $24(70.6 \%)$ & \multirow[t]{5}{*}{0.741} \\
\hline & LAD-LCX & $7(17.5 \%)$ & $13(16.3 \%)$ & & $6(17.6 \%)$ & $8(23.5 \%)$ & \\
\hline & LCX-OM & $2(5.0 \%)$ & $7(8.8 \%)$ & & $2(5.9 \%)$ & $1(2.9 \%)$ & \\
\hline & LAD-RI & $3(7.5 \%)$ & $1(1.3 \%)$ & & 0 & $1(2.9 \%)$ & \\
\hline & RCA-PD & $1(2.5 \%)$ & $3(3.8 \%)$ & & 0 & 0 & \\
\hline \multirow[t]{3}{*}{ Medina } & 1.1.1 & $31(77.5 \%)$ & $63(78.8 \%)$ & 1 & $26(76,5 \%)$ & $25(73.5 \%)$ & \multirow[t]{3}{*}{0.846} \\
\hline & 1.0 .1 & $5(12.5 \%)$ & $9(11.25 \%)$ & & $4(11.8 \%)$ & $6(17.6 \%)$ & \\
\hline & 0.1 .1 & $4(10.0 \%)$ & $8(10.0 \%)$ & & $4(11.8 \%)$ & $3(8.8 \%)$ & \\
\hline
\end{tabular}

Values are $\mathrm{n}(\%)$. LAD, left anterior descending; $\mathrm{D}$, diagonal artery; LCX, left circumflex; OM,obtuse marginal branch; RCA,right coronary artery; RI, ramus intermedius artery; PD, posterior descending artery

between the 2 groups in Tables 1 and 2.After propensity score matching, the incidence of immediate SB deterioration was significant lower in M-BSKT than PS [M-BSKT $n=6(17.6 \%) ; \quad$ PS $n=15(44.1 \%) ; \quad P=0.036$ ] (Table 3; Fig. 3a). Among them, there was no distinction about TIMI flow deterioration [M-BSKT $n=3(8.8 \%)$; PS $n=5(14.7 \%) ; P=0.709]$ and ostial pinching $[\mathrm{M}-\mathrm{BSKT} \mathrm{n}=$ $3(8.8 \%)$;PS $n=10(29.4 \%) ; P=0.062]$ between two groups. The application of rescue FKBI in M-BSKT was significant lower than PS [M-BSKT $\mathrm{n}=6(17.6 \%)$; PS $n=$ 15(44.1\%); $P=0.036$ ](Fig. 3b). In spite of all the patients who defined as SB deterioration underwent FKBI, 2 patients' SB were lost in PS group $[n=2(5.9 \%), P=$
0.492]. The prognosis at 12 months such as rehospitalization for unstable angina [M-BSKT $\mathrm{n}=3(8.8 \%)$; PS $\mathrm{n}=$ $1(2.9 \%)$ ] and MACEs [M-BSKT $n=0$; PS $\mathrm{n}=1(2.9 \%)$ ] were similar between the two groups $[P=0.613]$ showed in Table 3 and Fig. 4.

\section{Subgroup analysis of acute coronary syndrome (ACS)}

Baseline Characteristics and procedural outcomess of patients with acute coronary syndrome in the two groups were reanalyzed. The ACS patients were all recruited in two subgroups; 1$)$ M-BSKT group $(n=12)$ and 2) PS group $(n=33)$. There were no significant differences in baseline clinical characteristics between the

Table 3 Outcomes

\begin{tabular}{|c|c|c|c|c|c|c|}
\hline & \multicolumn{3}{|c|}{ pre propensity-matched analysis } & \multicolumn{3}{|c|}{ post propensity-matched analysis } \\
\hline & M-BSKT & PS & $P$ Value & M-BSKT & PS & $P$ Value \\
\hline & $n=40$ & $n=80$ & & $n=34$ & $n=34$ & \\
\hline \multicolumn{7}{|l|}{ immediate procedural and clinical outcomes } \\
\hline Device and procedural success & $40(100.0 \%)$ & $80(100.0 \%)$ & 1 & $34(100.0 \%)$ & $34(100.0 \%)$ & 1 \\
\hline Main-vessel TIMI flow 3 after procedure & $40(100.0 \%)$ & $80(100.0 \%)$ & 1 & $34(100.0 \%)$ & $34(100.0 \%)$ & 1 \\
\hline Side-branch deterioration & $7(17.5 \%)$ & $28(35.0 \%)$ & 0.076 & $6(17.6 \%)$ & $15(44.1 \%)$ & 0.036 \\
\hline SB TIMI flow <3 & $3(7.5 \%)$ & $12(15 \%)$ & 0.38 & $3(8.8 \%)$ & $5(14.7 \%)$ & 0.709 \\
\hline SB ostial pinching $\geq 90 \%$ & $4(10 \%)$ & $14(17.5 \%)$ & 0.412 & $3(8.8 \%)$ & $10(29.4 \%)$ & 0.062 \\
\hline SB occlusion immediately & 0 & $2(2.5 \%)$ & 0.552 & 0 & 0 & NS \\
\hline rescue FKBI & $7(17.5 \%)$ & $26(32.5 \%)$ & 0.129 & $6(17.6 \%)$ & $15(44.1 \%)$ & 0.036 \\
\hline Bailout stenting & 0 & $2(2.5 \%)$ & 0.552 & 0 & 0 & NS \\
\hline Revascularization & $7(17.5 \%)$ & $23(28.8 \%)$ & 0.263 & $6(17.6 \%)$ & $13(38.2 \%)$ & 0.104 \\
\hline SB loss & 0 & $5(6.3 \%)$ & 0.168 & 0 & $2(5.9 \%)$ & 0.492 \\
\hline Wire or balloon damaged & 0 & 0 & NS & 0 & 0 & NS \\
\hline \multicolumn{7}{|l|}{ The prognosis after 12 months } \\
\hline Stable condition & $37(92.5)$ & $73(91.3 \%)$ & 0.558 & $31(91.2 \%)$ & $32(94.1 \%)$ & 0.613 \\
\hline Rehospitalization for unstable angina & $3(7.5 \%)$ & $4(5.0 \%)$ & & $3(8.8 \%)$ & $1(2.9 \%)$ & \\
\hline MACES & 0 & $3(3.8 \%)$ & & 0 & $1(2.9 \%)$ & \\
\hline
\end{tabular}

Values are n(\%).TIMI, thrombolysis in myocardial infarction; FKBl, final kissing-balloon inflation; MACEs, major adverse cardiac events 

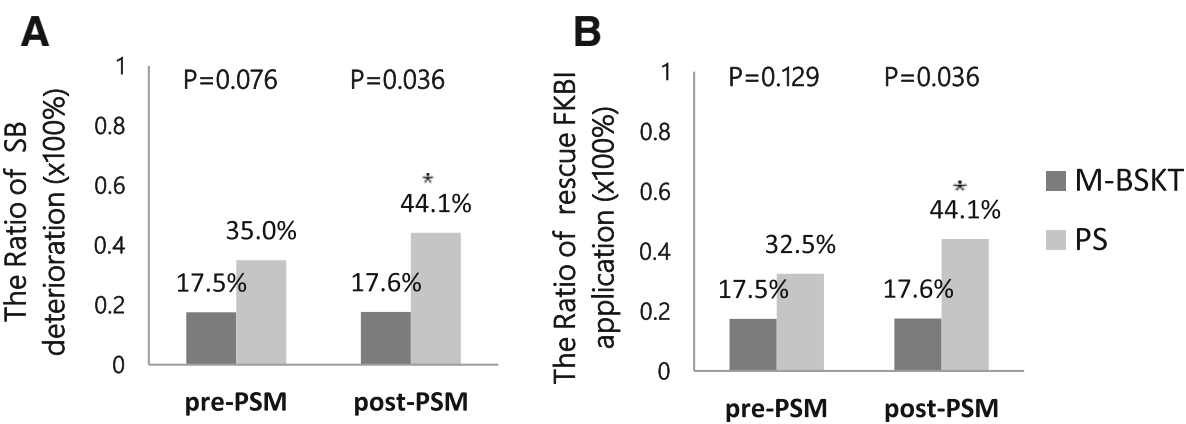

Fig. 3 a The Ratio of SB deterioration. The $P$-values of the incidence of immediate SB deterioration between the 2 groups (pre-PSM and postPSM). $\mathbf{b}$ The Ratio of rescue FKBI application. The $P$-values of the rescue FKBI application between the 2 groups (pre-PSM and post-PSM). PSM: propensity-matched analysis. FKBl: final kissing balloon inflation. ${ }^{*}$ means $P<0.05$

two groups. Angiographic and procedural characteristics of the two groups are shown in Table 4. The immediate SB deterioration in the PS group $[n=17(51.5 \%)]$ was significant higher compared to M-BSKT group [n = $1(8.3 \%) ; P=0.014]$ (Fig. 5a). All situations of SB deterioration were not identical between two groups (Fig. 5c). The PS group displayed a higher $(P=0.042)$ rate $[\mathrm{n}=$ $10(30.3 \%)]$ of SB TIMI flow $<3$ than M-BSKT. FKBI was more frequently performed in PS group $[\mathrm{n}=15(45.5 \%)]$ than M-BSKT group $[n=1(8.3 \%) ; P=0.033]$ (Fig. 5b). Despite the SB loss in two groups showed no difference, there were $4(12.1 \%)$ patients in ACS patients, which made a big proportion of all patients.

\section{Discussion}

The present study focus on clinical outcomes between M-BSKT and PS techniques for simple true bifurcation lesions stratified the criteria. The principal findings in this study are as follows: 1) In general, M-BSKT was similar to PS in every aspects except the damage of SB and the rate of FKBI application. 2) In ACS patients, performing M-BSKT was a significant factor to protect SB, especially in preventing the deterioration of TIMI flow and reducing the application of FKBI.

Although the ESC guidelines strongly recommends provisional SB stenting taking into consideration not only immediate but also long-term results of this strategy [22], abrupt closure of the SB may occur after MV stent implantation [12-14]. Meanwhile, SB occlusion occurred more frequently in patients with true bifurcation lesions than in those with non-true bifurcation lesions [23]. In the present clinical trial, the final outcomes of provisional stenting of bifurcation lesions were not associated with significant improvement $[15,24]$. In our study, we compared the safety and efficacy of PS and M-BSKT during the treatment of some especial bifurcation lesions. It adopted the criteria of true bifurcation lesions and eliminated the complex bifurcation lesions established by Chen [20]. Based on previous researches, we eliminated complex bifurcation lesions that may get the maximum benefit from 2-stent techniques. Since a proportion of patients with a severe big SB lesions (simple true bifurcation lesions) would remain ischemic after MV stenting, the jailed SB wire or

Figure4

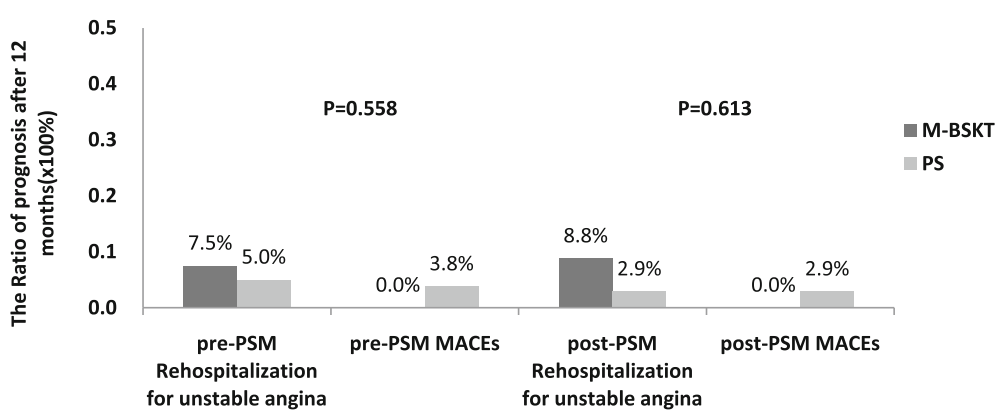

Fig. 4 Clinical outcomes at 12 months. PSM = propensity-matched analysis. Before PSM, 12 months clinical outcomes about rehospitalization for unstable angina and MACEs did not differ between patients treated with M-BSKT and PS. The prognosis at 12 months were similar between 2 groups after PSM 
Table 4 immediate procedural and clinical outcomes of ACS patients

\begin{tabular}{llll}
\hline patients with acute coronary syndrome & & & \\
\hline & M-BSKT & PS & $P$ Value \\
& $n=12$ & $n=33$ & \\
\hline immediate procedural and clinical outcomes & & \\
Device and procedural success & 12 & 33 & 1 \\
& $(100.0 \%)$ & $(100.0 \%)$ & \\
Main-vessel TIMl flow3 after & 12 & 33 & 1 \\
procedure & $(100.0 \%)$ & $(100.0 \%)$ & \\
Side-branch deterioration & $1(8.3 \%)$ & $17(51.5 \%)$ & 0.014 \\
SB TIMI flow <3 & 0 & $10(30.3 \%)$ & 0.042 \\
SB ostial pinching $\geq 90 \%$ & $1(8.3 \%)$ & $5(15.2 \%)$ & 1 \\
SB occlusion immediately & 0 & $2(6.1 \%)$ & 1 \\
rescue FKBI & $1(8.3 \%)$ & $15(45.5 \%)$ & 0.033 \\
Bailout stenting & 0 & $1(3.0 \%)$ & 1 \\
Revascularization & $1(8.3 \%)$ & $13(39.4 \%)$ & 0.07 \\
SB loss & 0 & $4(12.1 \%)$ & 0.561 \\
Wire or balloon damaged & 0 & 0 & NS \\
\hline
\end{tabular}

Values are $\mathrm{n}(\%)$.TIMI, thrombolysis in myocardial infarction; FKBI, final kissing-balloon inflation balloon facilitated rewiring of the SB by widening the angle between the MV and SB and prevented SB occlusion. This experiment using SB ostial pinching $\geq 90 \%$ by angiography as an indication for $\mathrm{SB}$ intervention reduced the unnecessary intervention of $\mathrm{SB}$ in the maximum limit. Meanwhile, a bailout stent in side branch was implanted only in case of dissection or significant flow impairment after FKBI.

The construction of the classical stent did not take into consideration vessel tapering in bifurcation lesions and resulted in carina and plaque shift-the main mechanisms of SB compromise [15]. However, the M-BSKT consists of leaving a dilated balloon in SB while implanting a stent in the MV. It impacts less on the bifurcation segment by means of limiting carina and plaque shift towards to $\mathrm{SB}$, which are two major factors responsible for SB compromise [18]. Yohei Numasawa [17] evaluated the configuration of true bifurcation lesion after stent implantation using the jailed balloon technique by three-dimensional optical frequency domain imaging (OFDI) which provided clearer and higher resolution images. It was already indicated clearly that there were no signs of plaque or carina shift into the SB. However, there was little data to support the advantage of this
A

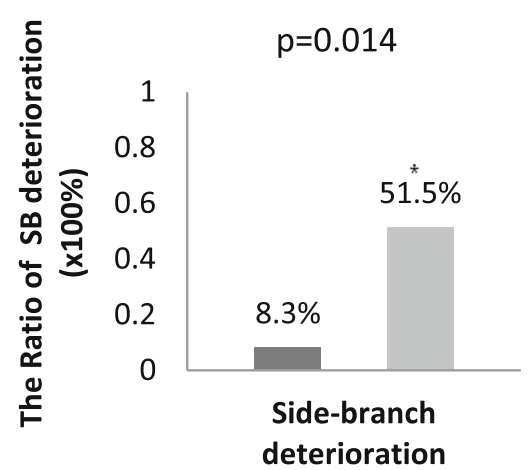

。

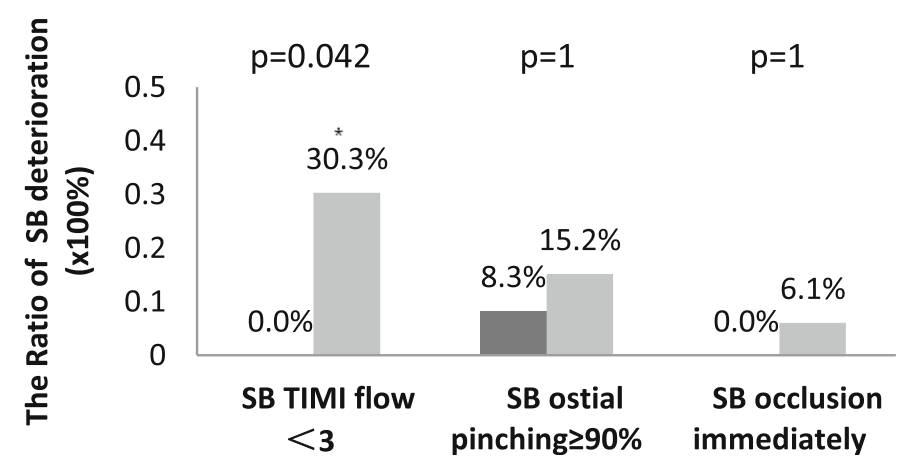

B

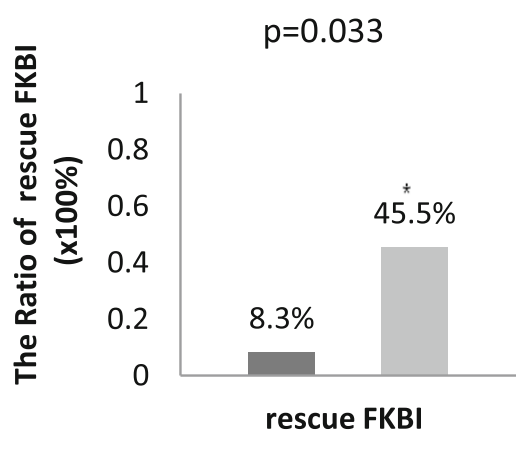

M-BSKT

PS

Fig. 5 immediate procedural and clinical outcomes of ACS patients. Subgroup analysis of ACS patients: (a) The ratio of immediate SB deterioration (b) The ratio of rescue FKBI application (c) The ratio of the each scenes of SB deterioration between the 2 groups. ${ }^{*}$ means $P<0.05$ 
technique. In this case, we clustered data from 2 groups about the SB ostial deteriorations and found out all deteriorations were slightly less when applying M-BSKT than PS. The advantage of M-BSKT that balloon inflated between the time from the stent located to inflated could protect SB more effective in theory.

Based on the date and trends we observed, the implementation of M-BSKT had no significant differences regarding to SB protection in the 2 study groups, but a trend towards superior clinical results in patients treated with M-BSKT. So the propensity score matching was used to identify the diversity between the 2 techniques. Baseline features were well balanced among M-BSKT patients versus PS patients. We noted the difference in immediate procedural and clinical outcomes between the 2 groups in the matched cohort. When propensity score matching was adopted, the effect of SB protection revealed the superior technical advantages of M-BSKT comparing with PS. As a result, the rate of SB flow deterioration and dissection after MV stenting at once was higher in PS. Because of cardiac death or myocardial infarction occurred more frequently in patients with SB occlusion [14], it is conceivable to infer that the inflated balloon of the SB provides a high degree of SB protection in immediate procedural.

According to research results available, jailed wire in SB was associated with the recovery of the occluded SB [14]. What's more, it indicated that M-BSKT used as a rescue procedure to keep the SB open was superior to PS on the basis of technological advantages, once the branch was occluded. This led to significantly reduced proximal deformation with related malapposed struts and reduced rate of SB ostial stenosis. Jailed balloon in SB could be inflated and FKBI could be applied, as long as the damage of SB flow happened accidentally. In some respect, $\mathrm{MB}$ restenosis was higher in the routine FKBI group due to the potential distortion of the MB stent strut [25]. On this premise, it is recommended to avoid deploying FKBI technique. The hypothesis that M-BSKT could decrease the proportion of FKBI implement during operation was justified in this study.

Interestingly, when we conducted subgroup analysis of patients with acute coronary syndrome, the advantages of M-BSKT were showed prominently. It just made a clarity conclusion that M-BSKT may provide more protection of SB. Patients with ACS are likely to have multiple vulnerable plaques that are liable to rupture or shift $[26,27]$. According to ACS patients with special bifurcation lesions, M-BSKT may limit thrombosis and plaque shift towards to SB more effectively, which avoid the impact on TIMI flow and reduce the application of FKBI.

As we all know, POT consists of an inflating balloon to the MV reference diameter which makes the malapposition in the stented MV segment completely corrected while maintaining perfect arterial circularity [19]. Though high rates of restenosis and stent thrombosis were still often observed after stenting, POT would provide potential benefits such as partially reducing malapposition and achieving effective modification of physiological anatomy $[19,28]$. In this study, POT was used as a favourable step to modify BSKT in order to reduce severe coronary flow problems.

Irrespective of propensity score matching, the findings of 1 year clinical follow-up were similar between the 2 techniques. However, wether M-BSKT will play a safe and effective role for a longer period of time is still unknown. Evaluation of long clinical outcomes of two stent percutaneous intervention strategy for treatment of coronary bifurcation lesions offered advantage over PS, simultaneously [11]. Therefore, with our present data, M-BSKT elicited more advantages than PS applied to simple true bifurcation lesions in the operation procedure.

\section{Limitations}

The first and foremost limitation of this study was that it did not involve a significant population. Also, visual estimation of the diameters was used in this study to dedicate bifurcations, rather than quantitative coronary measurement. It may be not precise for the selection of clinically relevant SB. Previous studies suggested that angiographic visual assessment of jailed SB lesions tended to overestimate the severity of jailed SB lesions compared to functional assessment by FFR. Regrettably, there was no application of FFR in this study. Differences in the angiographic assessment of SB lesion severity can affect the treatment strategy for bifurcation lesions. This manuscript used SB ostial pinching $\geq 90 \%$ by angiography as indication for SB intervention that was reduced the unnecessary intervention of $\mathrm{SB}$ in the maximum limit. Of note, there was a discrepancy between the assessment of the ischemia-inducibility and plan-to-treat. These variable and discrepant values might have influenced the differences of SB intervention between two groups. Although bifurcation angle was regarded as one of the important factors for bifurcation PCI, we excluded it from the scope of criteria, which may influence the SB-occlusion rate. In addition, fractional flow reserve for evaluating the significance of $\mathrm{SB}$ ostial lesions wasn't used before and after the procedure. Because randomization may interfere with the chosen of different bifurcations, the simple true bifurcations of the right coronary artery were rarely contained in our study.

\section{Conclusions}

We demonstrated that, for patients with coronary simple true bifurcation lesions, M-BSKT provides preferable 
outcomes comparing with the PS after propensity score matching. Because of the existence of vulnerable plaques in ACS patients exist, the advantages of M-BSKT were showed prominently. Our case reported that it appeared to be a reasonable and suitable strategy to reduce the deterioration of SB and the application of FKBI when MV stent was inflated during the operation. Although M-BSKT has been performed, its long-term impact on patients' outcomes cannot be ascertained based on current studies and requires further investigation on the correlation between BSKT and clinical outcomes.

\begin{abstract}
Abbreviations
ACS: Acute coronary syndrome; CABG: Coronary artery bypass graft; CKMB: Creatine kinase-MB; FFR: Fractional flow reserve; FKBI: Final kissing balloon inflation; MADEs: Major adverse cardiac events; M-BSKT: Modified balloon-stent kissing technique; MI: Myocardial infarction; MV: Main vessel; NSTEMI: Non-ST-segment elevation myocardial infarction; OFDI: Optical frequency domain imaging; PCI: Percutaneous coronary intervention; POT: Proximal optimization technique; PS: Provisional stenting; SB: Side branch; STEMI: ST-segment elevation myocardial infarction; TLR: Target lesion revascularization; TNI: Troponin I; TVR: Target vessel revascularization
\end{abstract}

\section{Acknowledgments}

The authors thank Dr. Bingchen Liu for insightful discussions throughout the study; and Dr. Xueqi Li for technical suggestions and support in conducting the experiments.

\section{Funding}

This study was supported by National Natural Science Foundation of China (Projects 81670381 and 81400710 to B.-C.L.).

\section{Availability of data and materials}

The datasets used and analysed during the current study are available from the corresponding author on reasonable request.

\section{Authors' contributions}

BC-L was responsible for the conception and design of the study. XQ-L provided the technical suggestions and support in conducting the experiments. WB-Q wrote the first draft and added important intellectual content. W-Z, JY$\mathrm{L}$ and $\mathrm{F}-\mathrm{Z}$ contributed to the data collection and analysis.SM-Z and $\mathrm{H}-\mathrm{T}$ modified the charts. SN-M and XQ-L contributed to refining the ideas and edited the manuscript. All authors contribute to critical revisions and agree to be accountable for all aspects of the work.

\section{Ethics approval and consent to participate}

Ethical approval was obtained from the Fourth Affiliated Hospital of Harbin Medical University Research Ethics Committee (2018-SCILLSC-01).

\section{Consent for publication}

Not applicable.

\section{Competing interests}

The authors declare that they have no competing interests.

\section{Publisher's Note}

Springer Nature remains neutral with regard to jurisdictional claims in published maps and institutional affiliations.

Received: 20 July 2018 Accepted: 18 March 2019

Published online: 08 April 2019

\section{References}

1. Bennett J, Dubois C. Coronary bifurcation lesions: is less more. J Thorac Dis. 2016;8(10):E1351-e4
2. Sawaya FJ, Lefevre T, Chevalier B, Garot P, Hovasse T, Morice MC, et al. Contemporary approach to coronary bifurcation lesion treatment. JACC Cardiovascular interventions. 2016;9(18):1861-78.

3. Steigen TK, Maeng M, Wiseth R, Erglis A, Kumsars I, Narbute I, et al. Randomized study on simple versus complex stenting of coronary artery bifurcation lesions: the Nordic bifurcation study. Circulation. 2006;114(18):1955-61.

4. Genereux P, Kumsars I, Lesiak M, Kini A, Fontos G, Slagboom T, et al. A randomized trial of a dedicated bifurcation stent versus provisional stenting in the treatment of coronary bifurcation lesions. J Am Coll Cardiol. 2015;65(6):533-43.

5. Ferenc M, Ayoub M, Buttner HJ, Gick M, Comberg T, Rothe J, et al. Longterm outcomes of routine versus provisional T-stenting for de novo coronary bifurcation lesions: five-year results of the bifurcations Bad Krozingen I study. Eurolntervention : journal of EuroPCR in collaboration with the Working Group on Interventional Cardiology of the European Society of Cardiology. 2015:11(8):856-9.

6. Colombo A, Bramucci E, Sacca S, Violini R, Lettieri C, Zanini R, et al. Randomized study of the crush technique versus provisional side-branch stenting in true coronary bifurcations: the CACTUS (coronary bifurcations: application of the crushing technique using Sirolimus-eluting stents) study. Circulation. 2009;119(1):71-8.

7. Hildick-Smith D, de Belder AJ, Cooter N, Curzen NP, Clayton TC, Oldroyd KG, et al. Randomized trial of simple versus complex drug-eluting stenting for bifurcation lesions: the British bifurcation coronary study: old, new, and evolving strategies. Circulation. 2010;121(10):1235-43.

8. Maeng M, Holm NR, Erglis A, Kumsars I, Niemela M, Kervinen K, et al. Longterm results after simple versus complex stenting of coronary artery bifurcation lesions: Nordic bifurcation study 5-year follow-up results. J Am Coll Cardiol. 2013;62(1):30-4.

9. Bittl JA. Treatment of bifurcation lesions: less is more. J Am Coll Cardiol. 2015;65(6):544-5.

10. Behan MW, Holm NR, de Belder AJ, Cockburn J, Erglis A, Curzen NP, et al. Coronary bifurcation lesions treated with simple or complex stenting: 5-year survival from patient-level pooled analysis of the Nordic bifurcation study and the British bifurcation coronary study. Eur Heart J. 2016:37(24):1923-8.

11. Nairooz R, Saad M, Elgendy IY, Mahmoud AN. Long-term outcomes of provisional stenting compared with a two-stent strategy for bifurcation lesions: a meta-analysis of randomised trials. Heart. 2017; 103(18):1427-34

12. Dou K, Zhang D, Xu B, Yang Y, Yin D, Qiao S, et al. An angiographic tool for risk prediction of side branch occlusion in coronary bifurcation intervention the RESOLVE score system (risk prEdiction of side branch OccLusion in coronary bifurcation interVEntion). JACC Cardiovascular interventions. 2015:8(1 Pt A):39-46.

13. Stone GW, Maehara A, Muller JE, Rizik DG, Shunk KA, Ben-Yehuda O, et al. Plaque characterization to inform the prediction and prevention of Periprocedural myocardial infarction during percutaneous coronary intervention: the CANARY trial (coronary assessment by near-infrared of atherosclerotic rupture-prone yellow). JACC Cardiovascular interventions. 2015;8(7):927-36.

14. Hahn JY, Chun WJ, Kim JH, Song YB, Oh JH, Koo BK, et al. Predictors and outcomes of side branch occlusion after main vessel stenting in coronary bifurcation lesions: results from the COBIS II registry (COronary Blfurcation stenting). J Am Coll Cardiol. 2013;62(18):1654-9.

15. Kang SJ, Mintz GS, Kim WJ, Lee JY, Park DW, Lee SW, et al. Preintervention angiographic and intravascular ultrasound predictors for side branch compromise after a single-stent crossover technique. Am J Cardiol. 2011;107(12):1787-93.

16. Cayli M, Seker T, Gur M, Elbasan Z, Sahin DY, Elbey MA, et al. A novelmodified provisional bifurcation stenting technique: jailed semi-inflated balloon technique. J Interv Cardiol. 2015;28(5):420-9.

17. Numasawa $Y$, Hase H, Yamazaki H, Tabei R, Kuno T, Kodaira M, et al. Threedimensional optical frequency domain imaging of a true bifurcation lesion after stent implantation using the jailed semi-inflated balloon technique. SAGE Open Med Case Rep. 2017:5:2050313×17724711.

18. Jin Z, Li L, Wang M, Zhang S, Chen Z, Shen Z. Innovative provisional stenting approach to treat coronary bifurcation lesions: balloon-stent kissing technique. The Journal of invasive cardiology. 2013;25(11):600-4.

19. Finet G, Derimay F, Motreff P, Guerin P, Pilet P, Ohayon J, et al. Comparative analysis of sequential proximal optimizing technique versus kissing balloon 
inflation technique in provisional bifurcation stenting: Fractal Coronary Bifurcation Bench Test. JACC Cardiovasc Interv. 2015;8(10):1308-17.

20. Chen SL, Sheiban I, Xu B, Jepson N, Paiboon C, Zhang JJ, et al. Impact of the complexity of bifurcation lesions treated with drug-eluting stents: the DEFINITION study (definitions and impact of complEx biFurcation leslons on clinical outcomes after percutaNeous coronary IntervenTIOn using drugeluting steNts). JACC Cardiovascular interventions. 2014;7(11):1266-76.

21. Medina A, Suarez de Lezo J, Pan M. A new classification of coronary bifurcation lesions. Rev Esp Cardiol. 2006;59(2):183.

22. Kolh P, Windecker S, Alfonso F, Collet JP, Cremer J, Falk V, et al. 2014 ESC/ EACTS guidelines on myocardial revascularization: the task force on myocardial revascularization of the European Society of Cardiology (ESC) and the European Association for Cardio-Thoracic Surgery (EACTS). Developed with the special contribution of the European Association of Percutaneous Cardiovascular Interventions (EAPCI). Eur I Cardiothorac Surg. 2014;46(4):517-92.

23. Park TK, Park YH, Song YB, Oh JH, Chun WJ, Kang GH, et al. Long-term clinical outcomes of true and non-true bifurcation lesions according to Medina classification- results from the COBIS (COronary Blfurcation stent) II registry. Circ J. 2015;79(9):1954-62.

24. Chen SL, Zhang JJ, Han Y, Kan J, Chen L, Qiu C, et al. Double kissing crush versus provisional stenting for left Main distal bifurcation lesions: DKCRUSHV randomized trial. J Am Coll Cardiol. 2017;70(21):2605-17.

25. Rahman S, Leesar T, Cilingiroglu M, Effat M, Arif I, Helmy T, et al. Impact of kissing balloon inflation on the main vessel stent volume, area, and symmetry after side-branch dilation in patients with coronary bifurcation lesions: a serial volumetric intravascular ultrasound study. JACC Cardiovasc Interv. 2013;6(9):923-31.

26. Saba L, Anzidei M, Marincola BC, Piga M, Raz E, Bassareo PP, et al. Imaging of the carotid artery vulnerable plaque. Cardiovasc Intervent Radiol. 2014;37(3):572-85.

27. Sudo M, Hiro T, Takayama T, lida K, Nishida T, Fukamachi D, et al. Tissue characteristics of non-culprit plaque in patients with acute coronary syndrome vs. stable angina: a color-coded intravascular ultrasound study. Cardiovasc Interv Ther. 2016;31(1):42-50.

28. Foin N, Secco GG, Ghilencea L, Krams R, Di Mario C. Final proximal postdilatation is necessary after kissing balloon in bifurcation stenting. Eurolntervention. 2011;7(5):597-604.

Ready to submit your research? Choose BMC and benefit from:

- fast, convenient online submission

- thorough peer review by experienced researchers in your field

- rapid publication on acceptance

- support for research data, including large and complex data types

- gold Open Access which fosters wider collaboration and increased citations

- maximum visibility for your research: over $100 \mathrm{M}$ website views per year

At $\mathrm{BMC}$, research is always in progress.

Learn more biomedcentral.com/submissions 